\title{
OsFPFL4 is Involved in the Root and Flower Development by Affecting Auxin Levels and ROS Accumulation in Rice (Oryza sativa)
}

Yaomin Guo ${ }^{\dagger}$, Qi Wu', Zizhao Xie, Bo Yu, Rongfeng Zeng, Qian Min and Junli Huang ${ }^{*}$ (1)

\begin{abstract}
Background: FPF1 (flowering-promoting factor 1) is one of the important family involved in the genetic control of flowering time in plant. Until now, limited knowledge concerning FPF1 family in rice has been understood.

Results: As a homologue of AtFPF1, FPF1-like protein 4 of rice (OSFPFL4) is expressed in various tissues of plants. The functions of OSFPFL4 in rice were investigated by the reverse genetics approaches. Plants overexpressing OsFPFL 4 have shorter primary root, more lateral roots and adventitious roots than wild type; however, RNA interference (RNAi) of OsFPFL4 significantly inhibits the growth of root system, and also delays the flowering time in rice. Interestingly, increased or repressed expression of OsFPFL4 leads to shrunken anthers and abnormal pollen grains. It is well recognized that auxin plays important roles in plant root and flower development, and the root elongation is also regulated by reactive oxygen species (ROS) homeostasis. Here, our results show that rice plants overexpressing OsFPFL4 accumulate more auxin in the shoot and root, whereas RNAi lines have less auxin than wild type. As expected, the transcript levels of genes responsible for auxin biosynthesis and polar transport are altered in these OsFPFL4 transgenic plants. As to ROS, slightly higher ROS levels were detected in overexpression root and inflorescence than the counterparts of wild type; however, the ROS levels were significantly increased in the RNAi lines, due to increased expression of ROS-producers and reduced expression of ROS-scavengers.

Conclusion: Our results reveal that OSFPFL4 is involved in modulating the root and flower development by affecting auxin and ROS homeostasis in rice plants. OsFPFL4 controls auxin accumulation via affecting auxin biosynthesis and transport, and also modulates ROS homeostasis by balancing ROS producing and scavenging. Thus, auxin-mediated ROS production might play a role in regulating redox status, which controls plant root and flower development.
\end{abstract}

Keywords: Auxin, Flower, OsFPFL4, Root, ROS

\section{Background}

Promoted root system architecture is crucial for crop growth performance and productivity (Van Norman et al. 2013). Most monocotyledonous plants have fibrous root systems composed of the adventitious root and lateral root (Jiang et al. 2017; Huang et al. 2018). Primary root development starts during embryogenesis. Lateral

\footnotetext{
* Correspondence: huangjunli@cqu.edu.cn; huang_junli@126.com ${ }^{\dagger}$ Yaomin Guo and Qi Wu contributed equally to this work. Key Laboratory of Biorheological Science and Technology (Chongqing University), Ministry of Education, Bioengineering College, Chongqing 400030, China
}

roots initiating from the primary root play critical roles in plant root development, since they determine the architecture of the root system and maximize the potential of the root system for water and nutrient uptake (Hochholdinger et al. 2004; Parizot et al. 2008; Coudert et al. 2010). As a post-embryonic mode of organogenesis in plant, development of lateral roots is a typical example of de novo organogenesis, according to a regular pattern (De Smet et al. 2006). Plant hormone auxin directs many developmental responses, including the elaboration of branching patterns in the root (Guseman 
et al. 2015). Endogenous auxin biosynthesis, polar transport, and degradation/conjugation can change its accumulation and auxin-dependent signaling (Petricka et al. 2012; Lavenus et al. 2013), and mutations involved in these processes significantly affect the lateral root development (Fukaki and Tasaka 2009; Peret et al. 2009). For instance, gain-of-function mutants such as yuccal-D, sur1, and sur 2 with increased auxin levels produce more lateral roots (Zhao et al. 2001; Peret et al. 2009). By contrast, mutants with altered auxin transport, such as Arabidopsis aux1, lax3, pin1, pin 3/7, pin 4/7 and rice osaux 1 have less lateral roots (Benkova et al. 2003; Peret et al. 2009; Zhao et al. 2015).

Although reactive oxygen species (ROS) such as superoxide anions $\left(\mathrm{O}_{2}^{-}\right)$and hydrogen peroxide $\left(\mathrm{H}_{2} \mathrm{O}_{2}\right)$ are generally considered to be toxic to cells, a series of evidence proved that ROS at appropriate levels function as ubiquitous signaling molecules to regulate plant development and stress adaptation (Xia et al. 2009; Ivanchenko et al. 2013). Specifically, ROS produced in the apoplast by NADPH oxidase, encoded by RESPIRATORY BURST OXIDASE HOMOLOGS $(R B O H s)$, play diverse roles in the regulation of seed germination (Mueller et al. 2009), development of pollen tubes (Potocky et al. 2007), and bud outgrowth (Chen et al. 2016). ROS also act a part in the root development. Recently, an increasing number of evidence showed that ROS function in the regulation of root meristem activity (Yang et al. 2014), root cell differentiation (Tsukagoshi et al. 2010), root elongation (Tsukagoshi et al. 2010; Chen et al. 2016; Liu et al. 2016), lateral root emergence (Orman-Ligeza et al. 2016), and root hair formation (Sundaravelpandian et al. 2013). In another report, PvRbohB silencing reduces the lateral root density in transgenic Phaseolus vulgaris roots (Montiel et al. 2013), implying that hormone-controlled developmental events are mediated by $R B O H s$ s. In addition, the pool of apoplast ROS can be modulated by the activity of class III peroxidases (PERs), which enable wall loosening by generating ROS (Passardi et al. 2004). It has been described that auxin-mediated developmental processes are closely associated with ROS production (Xia et al. 2015). Auxin-induced ROS are directly involved in cellwall loosening and have a crucial role in cell elongation (Schopfer 2001), and overexpression of the cell-walllocalized ascorbate oxidase gene results in increased oxidation of the apoplast and mimics auxin-mediated effects on plant growth (Pignocchi et al. 2003). Moreover, the increased cellular oxidation state associated with auxin maxima is thought to arrest the cell cycle in the quiescent center, which is important for the maintenance of the root meristem (Jiang and Zhang 2003; Heyman et al. 2013).
Flowering and floral development are very important traits for cultivars in agriculture since they impact crop yield. FLOWERING PROMOTING FACTOR 1 from mustard (MUFPF1) was originally understood based on its role in flowering, and overexpression of MuFPF1 promoted flowering time in Arabidopsis (Melzer et al. 1990; Kania et al. 1997). Up to now, homologous genes of MUFPF1 have been characterized in Arabidopsis (Melzer et al. 1999), rice (Oryza sativa) (Ge et al. 2004), tobacco (Nicotiana tabacum) (Smykal et al. 2004), and cotton (Gossypium L.) (Wang et al. 2014), which have been shown to confer promotion of flowering time. Similar to $M U F P F 1$, constitutive expression of AtFPF1 can lead to early flowering in Arabidopsis (Melzer et al. 1999). Additionally, introduction of AtFPF1 into rice also conferred early flowering, suggesting that it is involved in the genetic control of flowering time in both dicots and monocots (Xu et al. 2005). It is notable that rice plants overexpressing AtFPF1 also had more adventitious roots and shorter primary and adventitious roots than wild type (Xu et al. 2005). Overexpression of cotton GhFPF1 in Arabidopsis promoted flowering time and shadeavoidance responses (Wang et al. 2014). In rice, a homologue of AtFPF1, OsRAA1 (FPF1-like 1), has been characterized. Different from AtFPF1, OsRAA1 did not obviously modulate flowering time in rice, but was involved in auxin-mediated flower and root development (Ge et al. 2004). In summarize, FPF1 gene family takes part in several aspects of plant development. In spite of these progresses, limited knowledge concerning the underlying mechanism of FPF1 family in plant is understood.

In this report, we investigated the developmental roles of another novel FPF1 homologue in rice, OsFPFL4 (FPF1-like 4), by reverse genetics approaches. We propose that auxin-induced ROS production might play crucial roles in the developmental processes performed by OsFPFL4.

\section{Results \\ OsFPFL4 is a Homologue of FPF1}

There are five FPF1-like genes in rice (denoted as FPF1like 1-5) (Additional file 3: Figure S1). Here, OsFPFL4 (FPF1-like 4), a novel FPF1 homologue in rice, was studied. Multiple alignment of amino acid sequences of a series of FPF1 homologues, MuFPF1 from white mustard (Sinapis alba), AtFPF1 from Arabidopsis thaliana, OsRAA1 from rice (Oryza sativa), NtFPF1 from tobacco (Nicotiana tabacum), ZmFPF1 from maize (Zea mays) and GhFPF1 from cotton (G. hirsutum L.), revealed that OsFPFL4 shared high similarity to other FPF1 proteins (Fig. 1a). The result also indicated that there was at least one conserved domain, -LGWERY-, present in this small protein family (Fig. 1a). Phylogenetic analysis of 
(a)

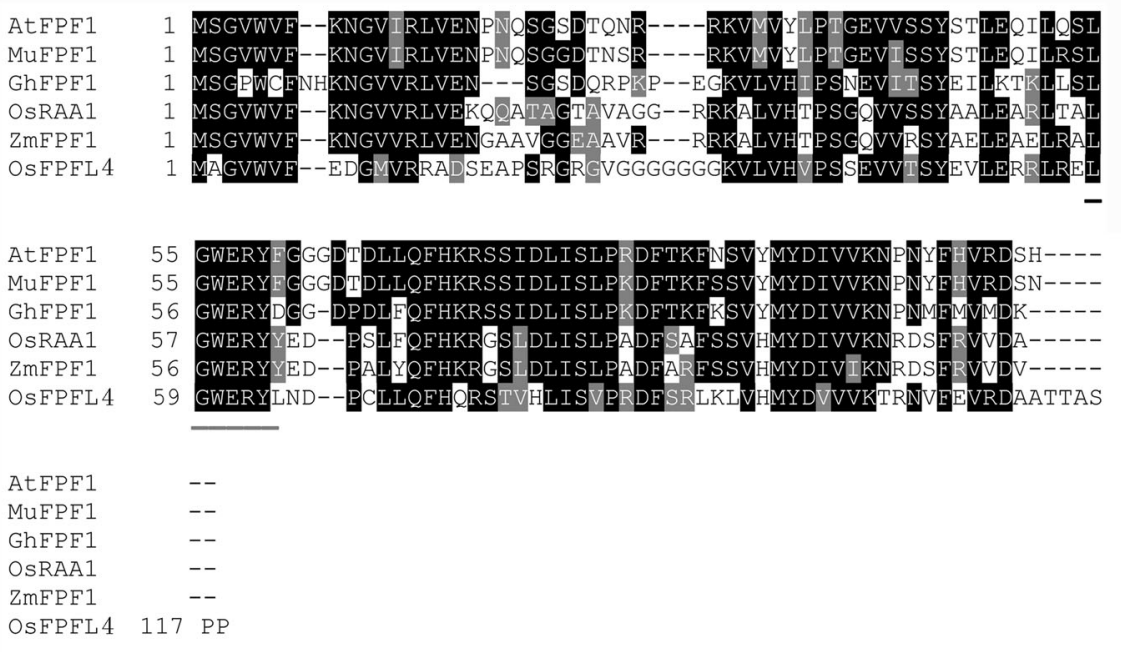

(b)

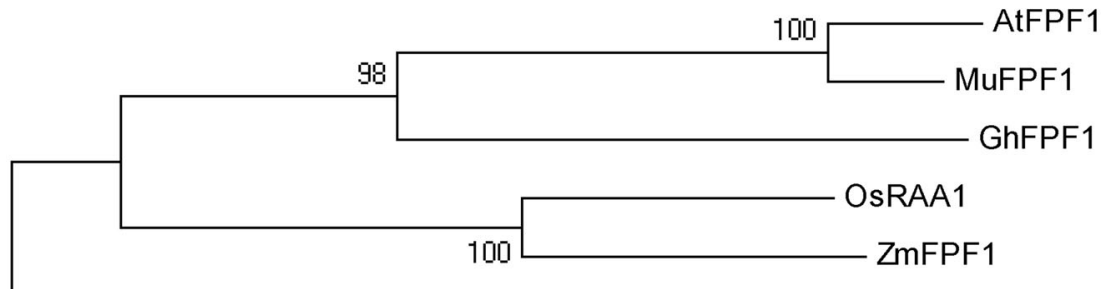

OsFPFL4

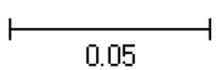

Fig. 1 Comparative analysis of OsFPFL4 and other FPF1 homologues. a Multiple alignment of FPF1 protein sequences in several species. AtFPF1 (Y11988) from Arabidopsis thaliana; MuFPF1 (Y11987) from mustard (Sinapis alba), NtFPF1 (AY496934) from (Nicotiana tabacum), ZmFPF1 (ACG44143) from maize (Zea mays), and OsRAA1 (AY659938) and OsFPFL4 (AK120187) from rice (Oryza sativa). b Phylogenetic tree of the FPF1 proteins in the above plants as determined by the MEGA 5.0 software package

FPF1 homologues indicated OsFPFL4 was located in a branch close to OsRAA1, but far from AtFPF1 (Fig. 1b), implying that OsFPFL4 might have functions similar to that of OsRAA1 in plant growth and development.

\section{Transcription Patterns of OsFPFL4}

To gain more insight into the genetic control of OsFPFL4 in plant development in rice, we examined its spatio- and temporal-transcription patterns. OsFPFL4 mRNA preferentially accumulated in the leaf blade and root at the seedling as well as tillering stages; however, at the heading stage, transcripts of OsFPFL4 peaked in the inflorescence, and only small quantities of transcripts accumulated in the leaf blade and leaf sheath (Fig. 2a). Scant transcripts of OsFPFL4 were detected in other organs except in young embryo at the ripening stage (Fig. 2a). These findings imply that OSFPFL4 might be involved in the growth and development of different organs of rice plants.

The responses of plant to environmental cues play an important role in adaption to the abiotic stress, and hormones are involved in the regulation of the developmental processes in plant. We therefore analyzed the response of OSFPFL4 in the seedlings treated by hormones or abiotic restraints. Remarkable response of OsFPFL4 to hormone IAA was observed, and the mRNA levels of OsFPFL4 were greatly induced by IAA (Fig. 2b). A significant increase in the transcription of OsFPFL4 was observed when rice seedlings were treated by $\mathrm{NaCl}$, mannitol or PEG (Fig. 2c-e), implying its roles in osmotic tolerance. Additionally, OsFPFL4 expression was also significantly induced in response to temperature stress (Fig. $2 \mathrm{f}$ and g). Overall, the expression patterns of OsFPFL4 indicate that it might be involved in plant growth and development and is therefore likely to be crucial for improving plant tolerance to external restraints.

OsFPFL4 is Involved in the Development of Root System and Plant Growth

To investigate the role of OsFPFL4 in plant growth and development in rice, we generated transgenic 


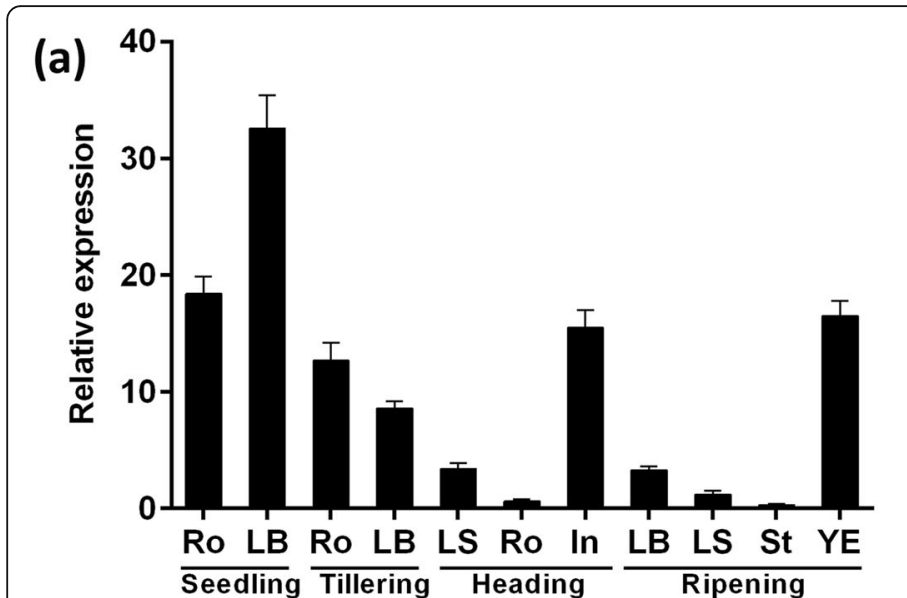

(c)

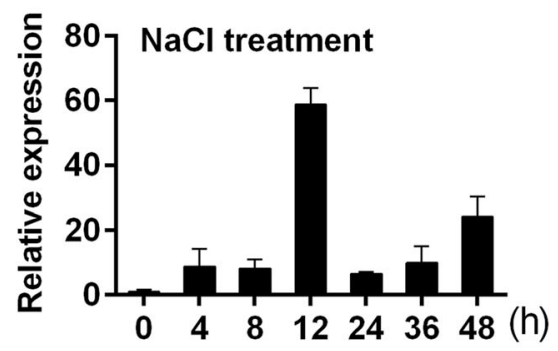

(b)

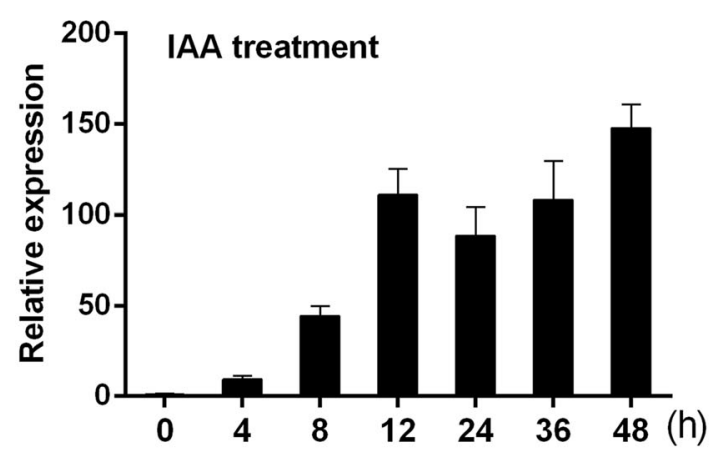

(e)
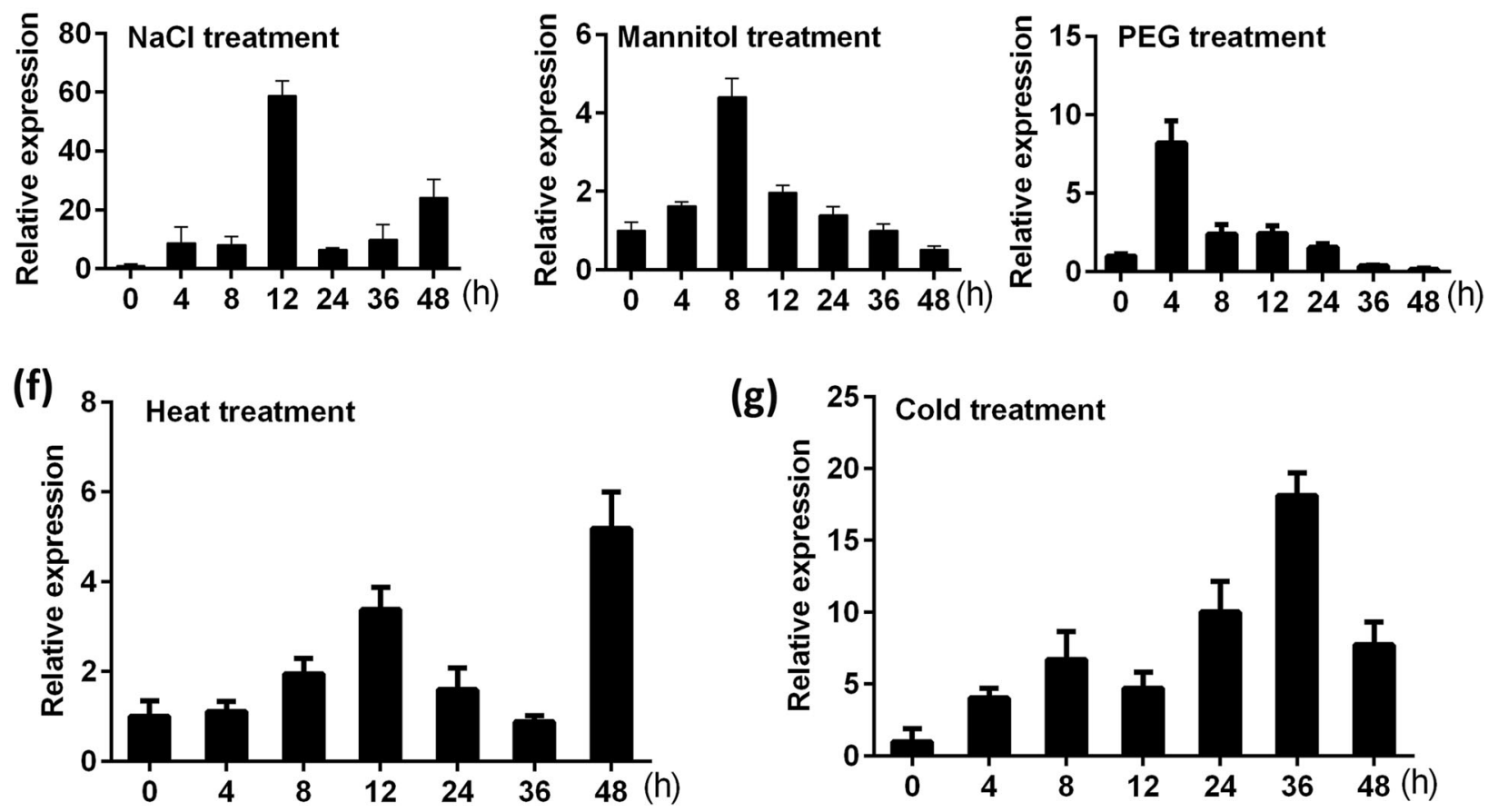

Fig. 2 Expression profiles of OsFPFL4 determined by quantitative PCR analysis. a Temporal and spatial expression patterns of OsFPFL4. Timecourses of OsFPFL4 expression in response to hormone of $20 \mu \mathrm{M}$ IAA (b) and abiotic stresses such as $200 \mathrm{mM} \mathrm{NaCl}$ (c), $100 \mathrm{mM}$ mannitol (d), $20 \%$ PEG6000 (e), heat $\left(42^{\circ} \mathrm{C}\right)(\mathbf{f})$ or cold $\left(4^{\circ} \mathrm{C}\right)(\mathbf{g})$, respectively, in 10-day-old seedlings. Seedlings grown in standard $1 / 2 \mathrm{MS}$ liquid medium under normal conditions were used as the control. Leaf samples were used for quantitative real-time PCR analysis. Error bars indicate standard error $(n=3)$. Three replica experiments were performed. Seedling, grown for 1 week; Tillering, grown for 1 month; Heading, grown for 2 months; Ripening, grown for 3 months. Ro, root; LB, leaf blade; LS, leaf sheath; In, inflorescence; St, stem; YE, young embryo

plants by overexpression or RNA interference (RNAi) of OsFPFL4, respectively (Fig. $3 \mathrm{a}$ and $\mathrm{b}$ ). The root system of both OsFPFL4-overexpression (OE-5 and OE-9) and -RNAi transgenic (RNAi-3 and RNAi-8) lines was changed greatly, compared with their wildtype counterparts after grown for 7 days in $1 / 2 \mathrm{MS}$ agar medium (Fig. 3a). Rice plants overexpressing OSFPFL4 exhibited promotion of the shoot growth as well as lateral root and adventitious root formation, whereas RNAi lines had the phenotype of repressed root and shoot growth (Fig. 3a). Compared to wild type, both OsFPFL4-overexpression and -RNAi seedlings showed markedly reduced primary root length (Fig. 3d). The shoot length, lateral root density and adventitious root number in overexpression plants were greatly increased, but the counterparts of RNAi plants were reduced significantly (Fig. $3 c$, e and f). In addition, up- or down-regulation of OsFPFL4 reduced 


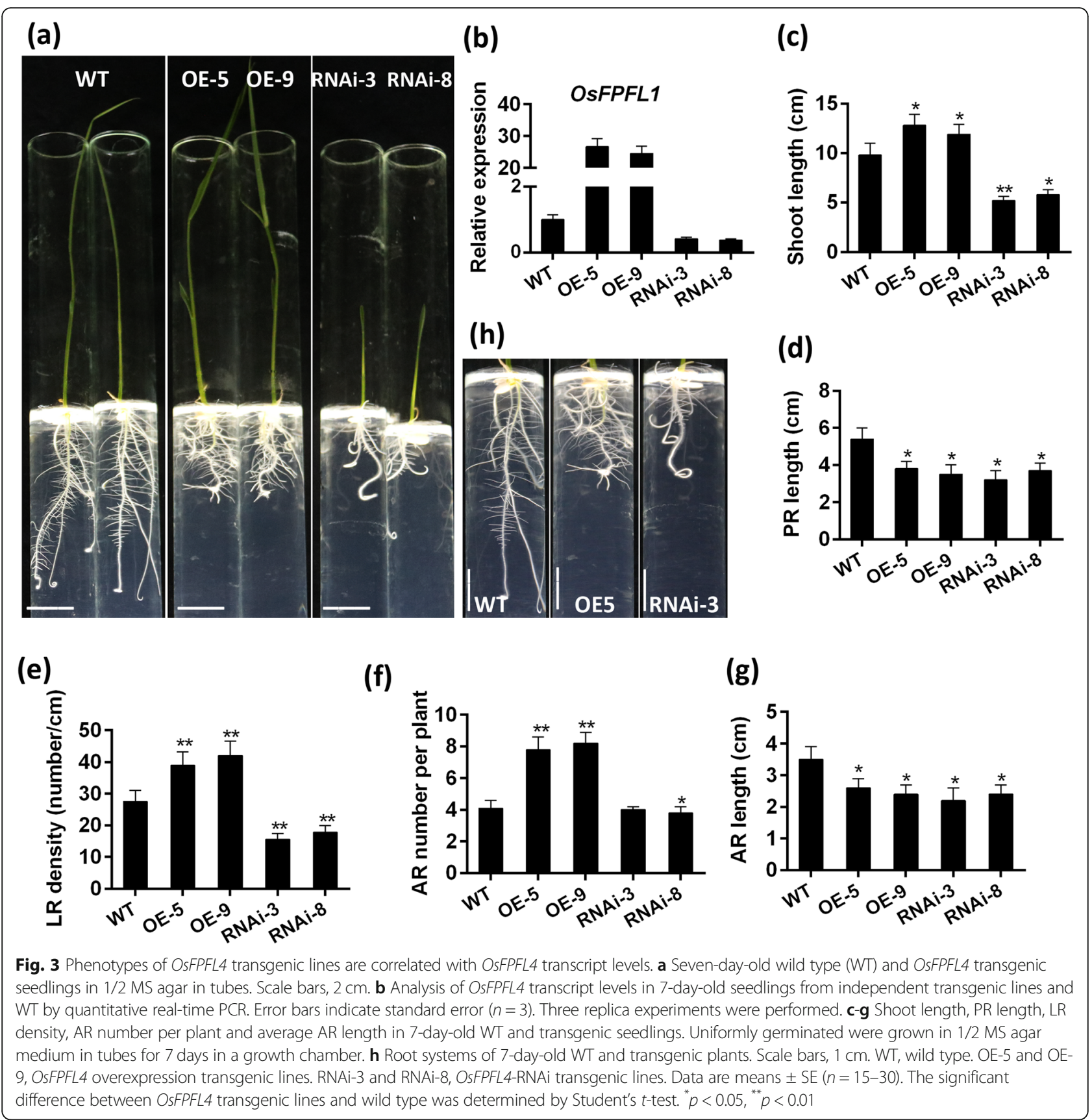

the average length of adventitious root (Fig. 3g). Although the length of lateral root was not quantified, it was clearly observed to be reduced in RNAi lines compared with that of wild type (Fig. 3h). It is notable that overexpression lines exhibited helix primary root (Fig. 3a). In comparison with wild-type plants grown for 60 days, the plants overexpressing OsFPFL4 displayed better performance with increased plant height and flag leaf length, whereas the growth of RNAi plants was remarkably inhibited (Fig. 4a-d). There seems to be no significant difference in the length of root system between wild type and transgenic lines grown in soil for 60 days, but the overexpression plants had more branched root system than wild type and RNAi lines (Fig. 4e). Accordingly, the biomass of root system was significantly enhanced in overexpression plants, whereas reduced greatly in RNAi plants, compared that of wild type (Fig. 4f), which might be caused by the difference of adventitious root number and lateral root density. Together, these results indicate that OsFPFL4 plays a key role in the root growth and development in rice. 


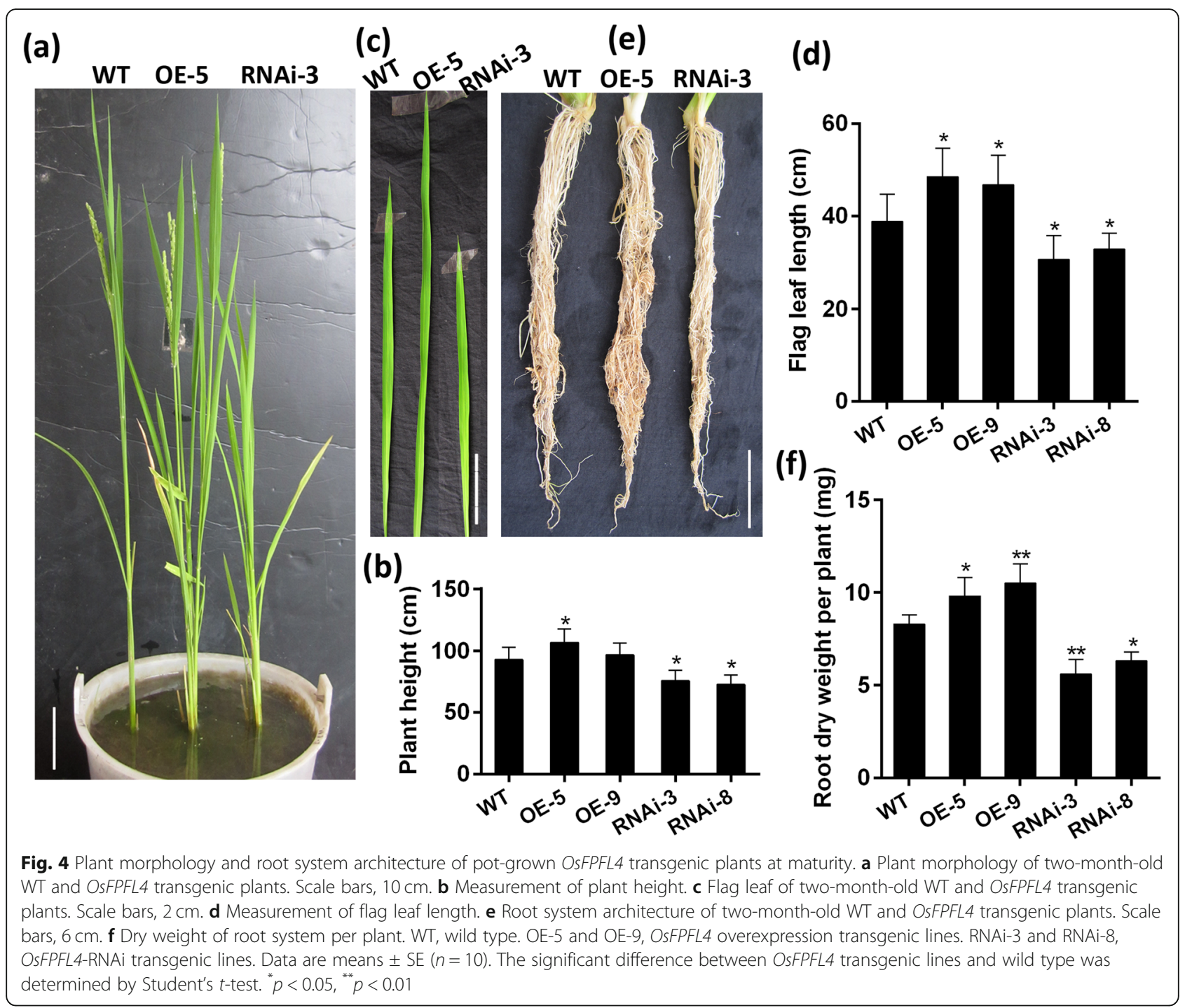

\section{Abnormal Expression of OsFPFL4 Affects the \\ Development of Anther and Pollen}

Considering that OsFPFL4 is preferentially expressed in inflorescence at heading stage (Fig. 2a), we then explored the role of OsFPFL4 in the control of flowering time and flower organ development. As shown in Fig. 4a, overexpression of OsFPFL4 did not obviously lead to early flowering in rice; however, RNAi lines exhibited apparently delayed flowering. As to flower organ development, there seemed to be no visible difference in the pistil between wild type and transgenic plants; however, abnormal expression of OSFPFL4 caused defective development of stamens (Fig. 5). The yellow and plump anthers appeared in wild-type plants at the flowering stage (Fig. 5a). Conversely, stamens of the OsFPFL4-overexpression or -RNAi plants showed obvious defects and were also smaller than the counterparts of wild type (Fig. 5b and c). Compared to wild type, up-regulation of
OsFPFL4 resulted in slightly shrunken anthers, whereas down-regulation of OSFPFL4 led to white and shrunken anthers more severely (Fig. 5a-c). We further observed the pollen development on day 5 after flowering. In comparison with wild type, a great number of abnormal pollens were observed in OsFPFL4-overexpression or RNAi plants, and the fertility of mature pollen in the RNAi lines seemed to be damaged more severely, indicated by $1 \% \mathrm{I}_{2}$-KI staining (Fig. $5 \mathrm{a}-\mathrm{c}$ ). Mature pollens of wild type were black and round in $\mathrm{I}_{2}-\mathrm{KI}$, while pollens from OSFPFL4-overexpression or RNAi plants at the same development period were grey and shriveled, or even broken (Fig. 5a-c), suggesting the pollen vitality might be severely impaired. Further investigation showed that the ratio of normal pollen of transgenic lines was remarkably reduced in overexpression or RNAi lines, compared to that of wild type (Fig. 5d). Together, these results indicate that normal expression of OsFPFL4 


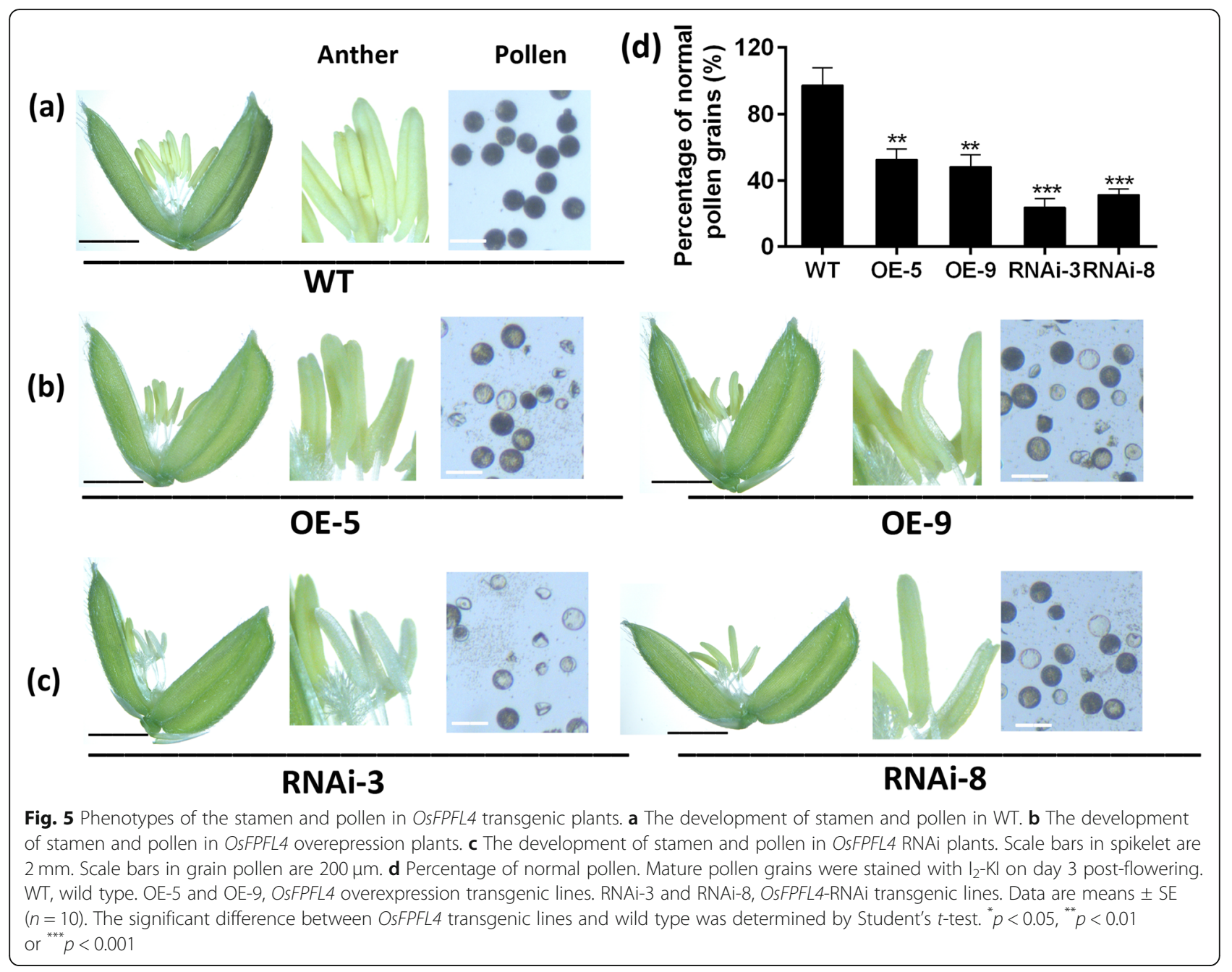

is required for the development of stamen and pollen in rice, implying its crucial roles in the grain yield.

\section{OsFPFL4 is Involved in the Root Development by Modulating Auxin Homeostasis}

It has been reported that the primary root and lateral root respond to auxin differently, and exogenous auxin can promote the lateral root development but reduce primary root elongation (Marin et al. 2010; Yoon et al. 2014). The phenotypes of increased lateral root density and inhibited primary root elongation were observed in OsFPFL4 overexpression plants (Fig. 3d and e), implying that endogenous auxin accumulation or polar auxin transport might be altered in OsFPFL4 transgenic plants. To determine whether the alteration of lateral root density and primary root length in transgenic plants was associated with endogenous auxin levels in roots, the IAA content was investigated in the overexpression, RNAi and wild-type seedlings by HPLC-MS-MS analysis. As shown in Fig. 6a, IAA content was significantly increased in roots of overexpression plants, whereas drastically reduced in RNAi plants, compared to that in wild type. In fact, enhanced auxin accumulation was also found in shoots of overexpression plants, whereas reduced auxin content was detected in RNAi shoots (Fig. 6b). Auxin levels in the roots are determined by local auxin biosynthesis, polar auxin transport, auxin breakdown, and/or auxin conjugation. We then investigated the transcript levels of auxin biosynthesis genes (OsYUCs), polar auxin transport genes (OsPINs and OsAUX/LAXs), and auxin inactivation genes (OsDAO and OsGH3s) in the roots. The results showed that OsYUC1, and OsYUC4 and OsYUC6 were found to be greatly up-regulated in overexpressing plants, whereas down-regulated in RNAi plants (Fig. 6c). As to OsPINs and OsAUX/LAXs, the transcript levels of OsPIN1a, OsPIN1b, OsPIN5a, OsPIN8, OsAUX1 and OsAUX2 were found to be drastically increased in overexpressing plants, but reduced in RNAi plants (Fig. 6d and e). Other genes involved in auxin biosynthesis and polar auxin transport did not show significant difference in transcript levels between transgenic lines and wild type (Fig. 6d and e). 


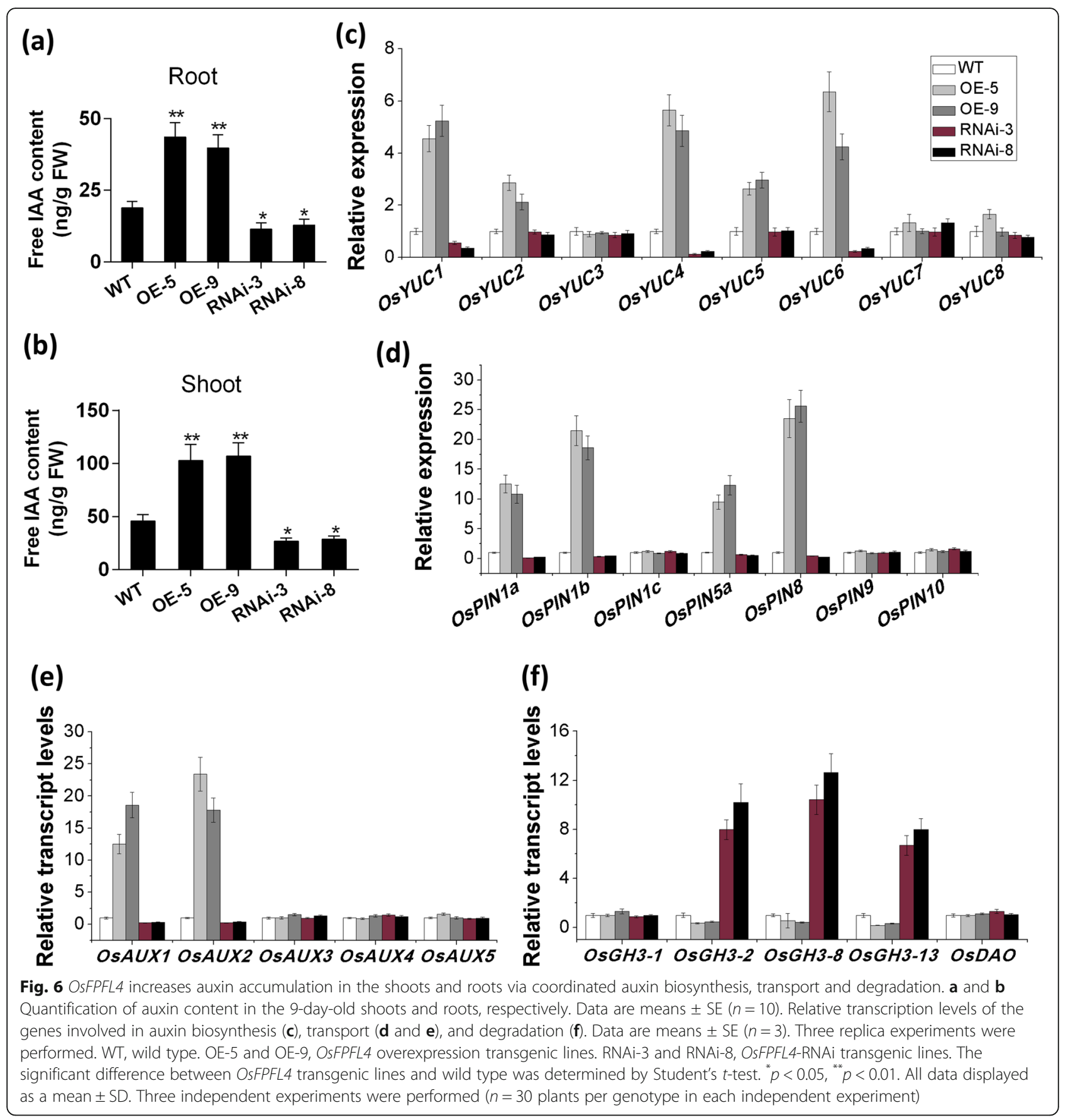

Transcription of OsGH3-2, OsGH3-8 and OsGH3-13 was greatly increased in RNAi plants, but there is no difference in OsDAO expression between transgenic lines and wild type (Fig. 6f).

\section{Abnormal Expression of OsFPFL4 Enhances ROS Levels}

The action of auxin in the regulation of root development is closely associated with ROS (Zhang et al. 2014a; Xia et al. 2015). To test whether the roots of OsFPFL4 transgenic lines have altered ROS levels, we used the
ROS-reactive dyes DAB and NBT to detect the levels of $\mathrm{H}_{2} \mathrm{O}_{2}$ and $\mathrm{O}_{2}^{-}$, respectively, in the primary root tip (Fig. 7a). As shown in Fig. 7b, the root tips of both OsFPFL4-overexpression and -RNAi lines had the stronger staining than that of wild type, and OsFPFL4-RNAi roots had the strongest staining. Compared to the strong staining just centralized in the first $1 \mathrm{~mm}$ of the root tip in wild type, the staining intensity continued to be strong in the differentiation zone and elongation zone of the root tip of OsFPFL4-RNAi plants (Fig. 7b). 
(a)

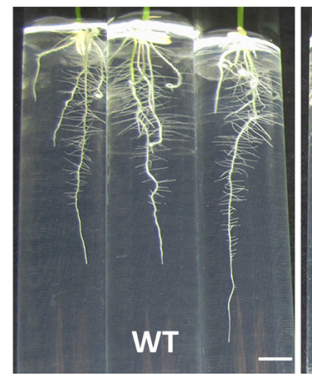

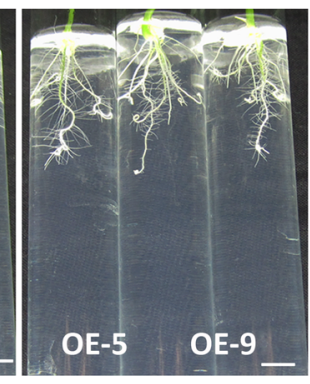

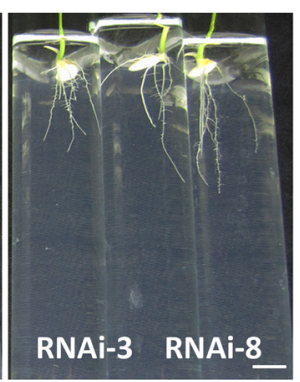

(b) $\mathrm{WT} \quad \mathrm{OE}$

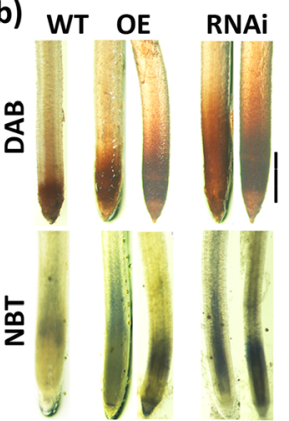

(c)

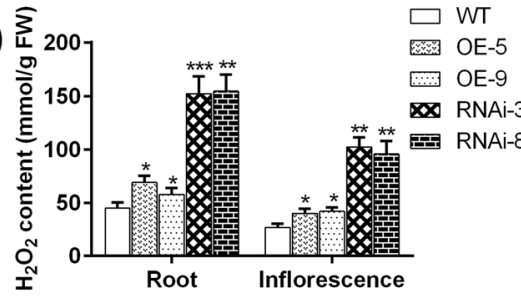

(f)

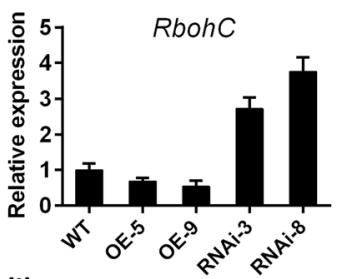

(i)

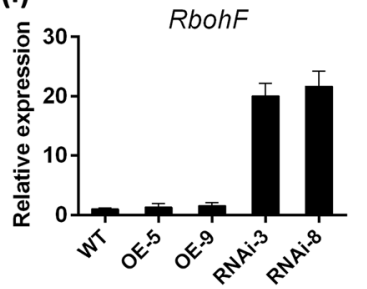

(d)

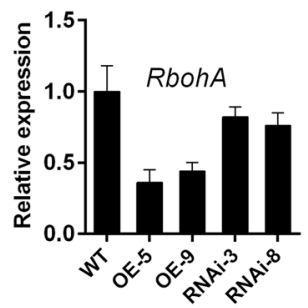

(e)

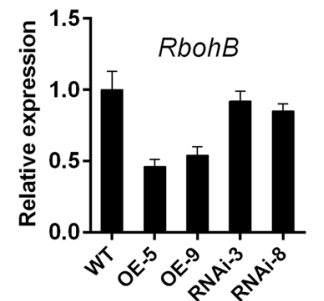

(g)

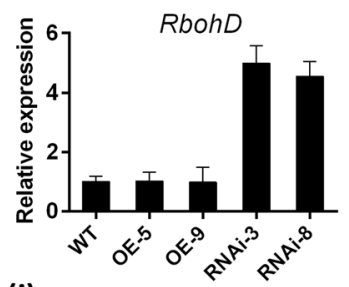

(j)

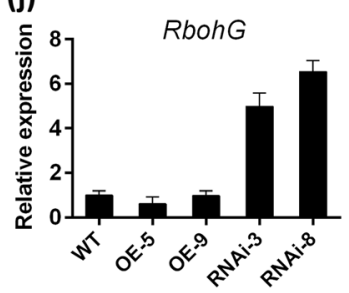

(h)

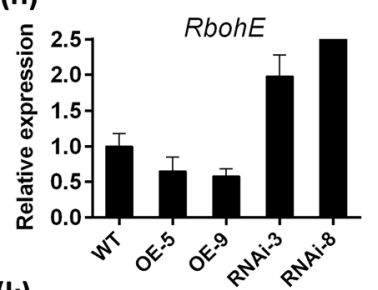

(k)

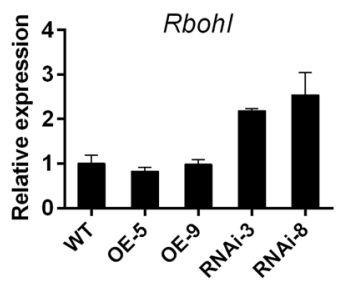

Fig. 7 OSFPFL4 alters ROS accumulation in the root and inflorescence through affecting the expression of ROS-producer Rbohs. a Root system architecture of 7-day-old seedling in $1 / 2 \mathrm{MS}$ medium. Scale bars, $1 \mathrm{~cm}$. b NBT and DAB staining for $\mathrm{O}_{2}{ }^{-}$and $\mathrm{H}_{2} \mathrm{O}_{2}$, respectively, in the root tips of wild type and OsFPFL4 transgenic lines. Scale bars, $1 \mathrm{~mm}$. c Quantification of $\mathrm{H}_{2} \mathrm{O}_{2}$ content in the roots of wild type and OsFPFL4 transgenic plants. Data are means \pm SE $(n=10)$. $\mathbf{d}$-kTranscript levels of OsRbohs in the roots of wild type and OsFPFL4 transgenic plants by qPCR analysis. Data are means \pm SE $(n=3)$. Three replica experiments were performed. WT, wild type. OE-5 and OE-9, OsFPFL4 overexpression transgenic lines. RNAi-3 and RNAi-8, OsFPFL4-RNAi transgenic lines. Data are means \pm SE $(n=10)$. The significant difference between OsFPFL4 transgenic lines and wild type was determined by Student's $t$-test. ${ }^{*} p<0.05,{ }^{* *} p<0.01$ or ${ }^{* * *} p<0.001$

Investigation of $\mathrm{H}_{2} \mathrm{O}_{2}$ content showed that its accumulation was slightly increased in OsFPFL4-overexpression roots but drastically enhanced in RNAi roots (Fig. 7c). ROS accumulation was also enhanced in inflorescence in transgenic plants (Fig. 7c). The apoplastic ROS are mainly produced by plasma membrane localized NADPH oxidases (Kadota et al. 2015). Thus, we further evaluated the transcription of ROS producers, Rbohs. As shown in Fig. $7 \mathrm{~d}-\mathrm{k}$, the transcript levels of most of OsRbohs were clearly reduced in OsFPFL4 overexpression roots, but significantly enhanced in OsFPFL4-RNAi roots, compared to that in wild type. These results suggest that OsFPFL4 might alter the expression of these ROS-producers to affect ROS levels.

In order to understand the functions of OsFPFL4 in the growth and development in rice plants in depth, global gene expression analysis of OSFPFL4 transgenic plants and wild type by RNA sequencing (RNA-Seq) was performed. As shown in Fig. 8a and b, there were a total of 2362 differentially expressed genes (DEGs) $(P<0.05)$ between OsFPFL4 overexpression and RNAi lines. Go Analysis showed that these DEGs are involved in various biological process such as growth and development, response to stimuli, signal transduction and cell apoptosis 
(a)

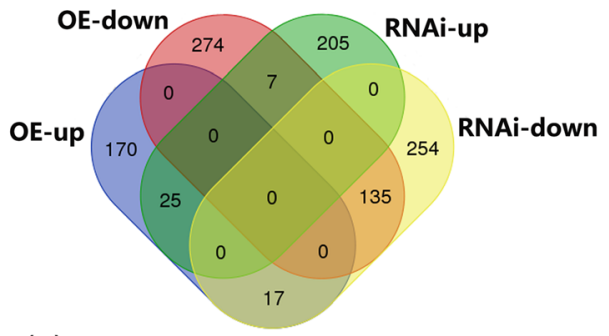

(c)

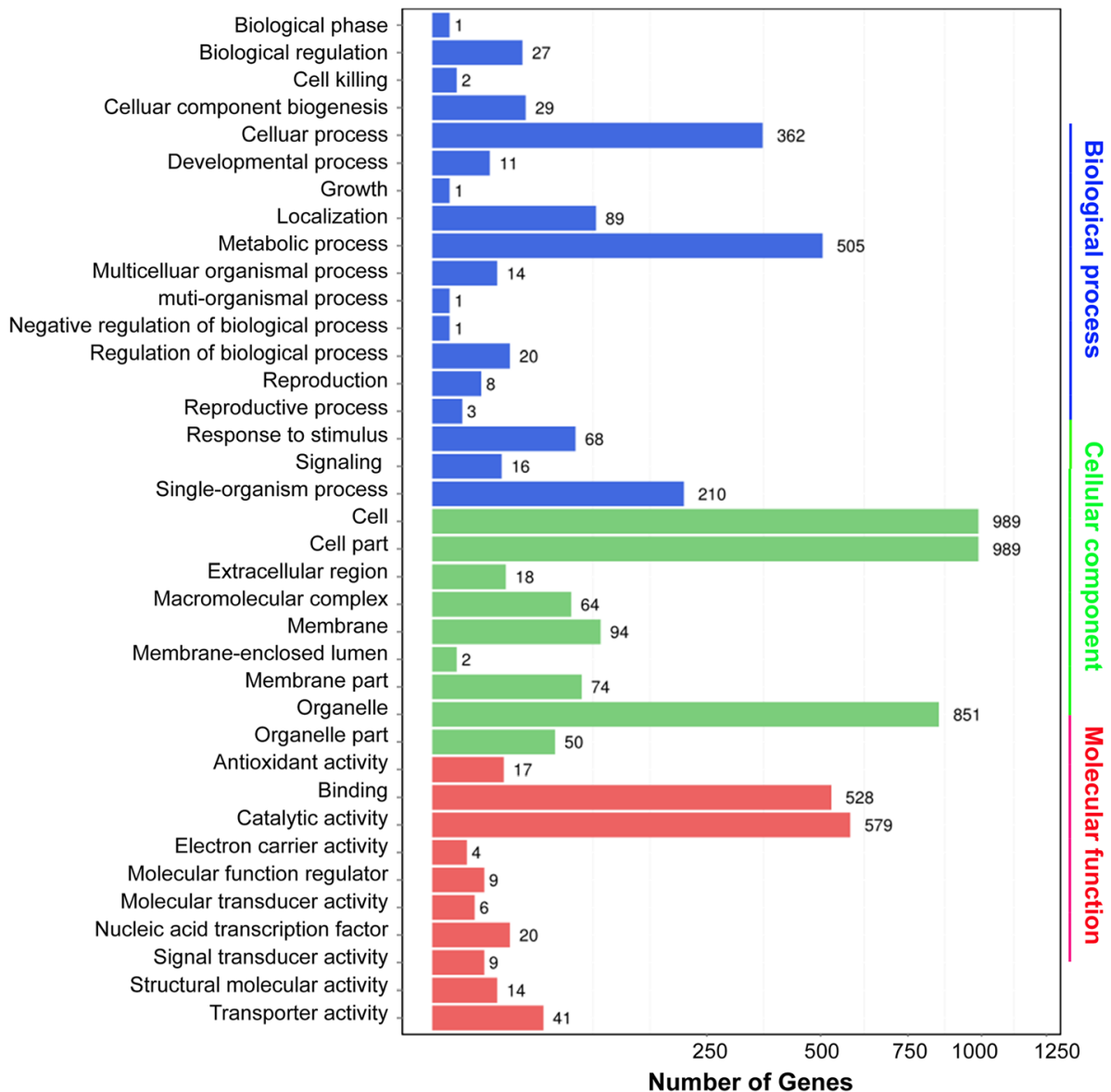

Fig. 8 Analysis of RNA-Seq data of wild type, OsFPFL4 overexpression and -RNAi transgenic lines. a Venn' plot of differentially expressed genes (DEGs) between wild type and OSFPFL4 transgenic lines. b The number of DEGS between wild type and OsFPFL4 transgenic lines. c Functional categorization of the DEGs between wild type and OsFPFL4 transgenic lines based on Gene Ontology (GO) annotation. Seedlings were grown in 1/2 MS medium for 7 days, and three biological replicates were used. LOC_Os01g27340, LOC_Os10g38720, LOC_Os03g17480, LOC_Os03g57200, LOC_Os10g38740, LOC_Os09g20220 are genes for glutathione S-transferases; LOC_Os07g01420, LOC_Os01g18950, LOC_Os07g44480, LOC_Os01g18970, LOC_Os10g39160, LOC_Os07g44460, LOC_Os07g31610, LOC_Os03g25320, LOC_Os06g16350, LOC_Os03g25280 are genes for peroxidases

(Fig. 8c). In plants, ROS homeostasis is strictly controlled by a delicate balance between ROS-producing and -scavenging enzymes (Qi et al. 2017). To our interesting, RNA-Seq analysis showed that the expression levels of multiple genes for ROS-scavenging enzyme glutathione $S$-transferase were increased in OsFPFL4 overexpression plants but reduced in RNAi lines

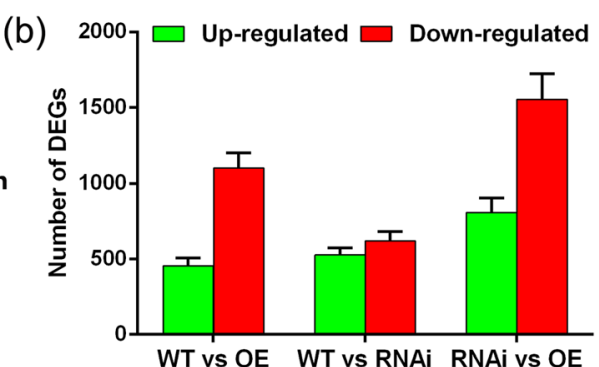



(Additional file 2: Table S2). The transcription of many peroxidases, which are considered as bifunctional enzymes that can scavenge $\mathrm{H}_{2} \mathrm{O}_{2}$ but also produce ROS, were also altered in transgenic lines (Additional file 2: Table S2). To validate the expression profiles obtained by RNA-Seq analysis, qPCR analysis was performed for these genes in Additional file 2: Table S2. As expected, 
qPCR analysis displayed similar patterns as the RNA-Seq data, despite some of quantitative differences in expression levels (Fig. 9). The results suggest that abnormal expression of OsFPFL4 might affect ROS levels by changing the transcription of genes for ROS-producing and -scavenging enzymes in rice plants.

\section{Discussion}

OsFPFL4 belongs to a small protein family that have no introns in their genomic sequences and was involved in the genetic control of flowering time in plant, denoted as FPF1 (Kania et al. 1997; Melzer et al. 1999; Ge et al. 2004; Wang et al. 2014). MuFPF1 was firstly studied as a floweringpromoting factor in mustard (Melzer et al. 1990), and its overexpression in Arabidopsis led to early flowering (Kania et al. 1997). AtFPF1 was proved to modulate flowering time via the GA-dependent signaling pathway in Arabidopsis (Melzer et al. 1999), and overexpression of AtFPF1 confers promotion of flowering time as well as formation of adventitious root in rice (Xu et al. 2005). OsRAA1, a homologue of FPF1, shared a 58\% sequence homology of amino acids with AtFPF1, and its overexpression caused pleiotropic phenotypes in transgenic rice plants, including altered leaf shape, flower and root development as well as root response to gravity (Ge et al. 2004). It is notable that, different from other FPF1s, overexpression of OsRAA1 did not promote flowering time, but caused abnormal florets with longer filaments and shrunken anthers (Ge et al. 2004). In our study, overexpression of OsFPFL4 did not obviously lead to early flowering in rice; however, RNAi lines exhibited delayed flowering (Fig. 4a), and OsFPFL4 transgenic lines had normal florets (Fig. 5a-c). There are a few genes involved in the root as well as flower development. MADS-box genes that are known to regulate the network of flower development also work in signal transduction in root development (Zhang and Forde 1998; Yu et al. 2017; Zhang et al. 2018). Similar to OsRAA1, OsFPFL4 was also indicated to modulate root development. Overexpression of OSFPF4 significantly reduced the primary root length, but greatly increased the lateral root density and adventitious root number (Fig. 3e and f), suggesting that there is similarity in the genetic control of flowering and root development between OSFPFL4 and OSRAA1.

The lateral root density were remarkably increased in OsFPFL4 overexpression lines, whereas reduced in RNAi lines (Fig. 3e), indicating that OsFPFL4 promotes formation of the lateral root. Increased auxin accumulation and transport can enhance lateral root formation (Peret et al. 2009; Zhao et al. 2015). In this study, we showed that OsFPFL4 promoted lateral root development by
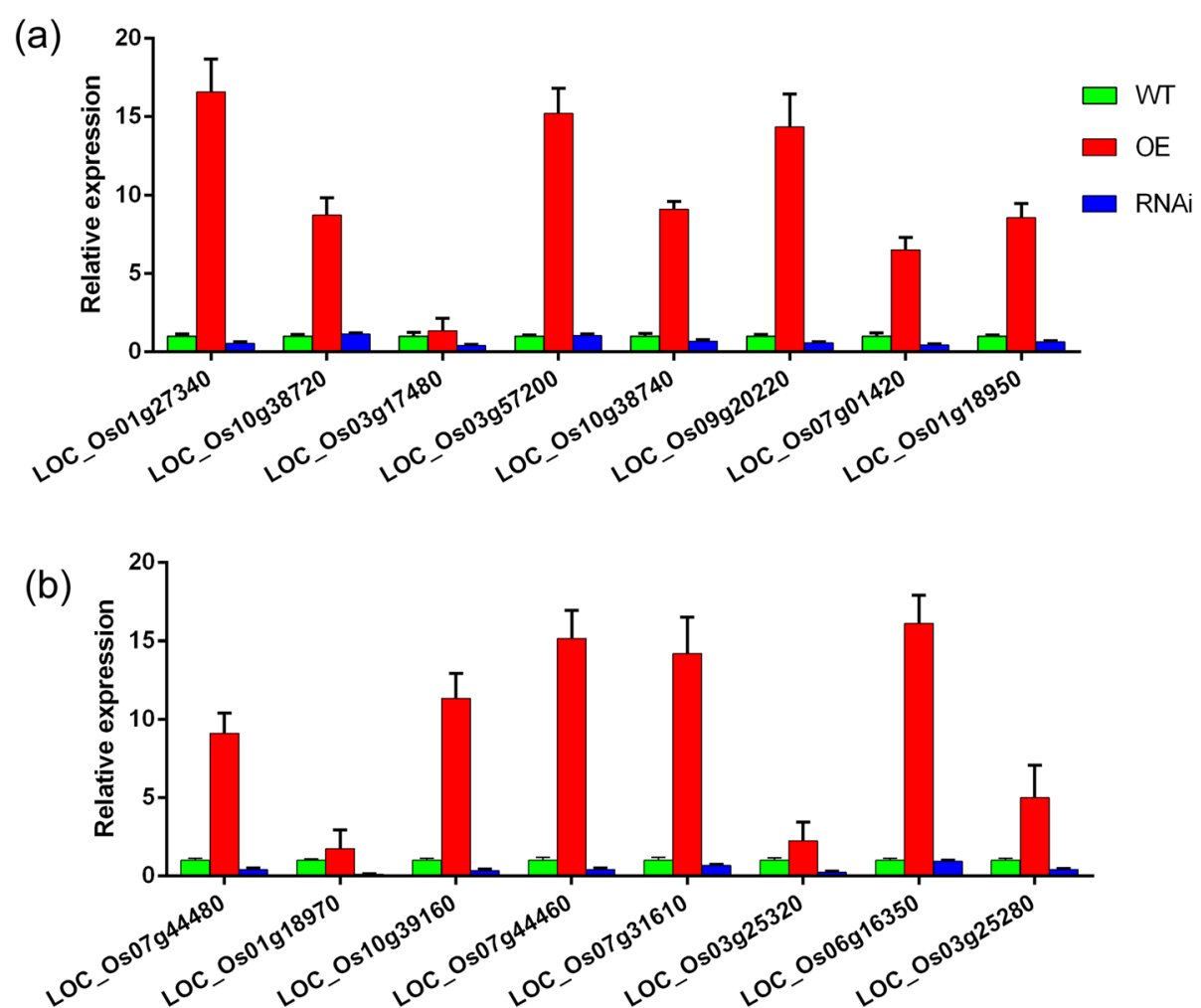

Fig. 9 Transcript levels of ROS-scavengers in wild and OsFPFL4 transgenic plants by quantitative PCR analysis. Seedlings were grown in 1/2 MS medium for 7 days. The data represent the means $( \pm \mathrm{SE})$ of three biological replicates. Three replica experiments were performed. WT, wild type. OE-5 and OE-9, OsFPFL4 overexpression transgenic lines. RNAi-3 and RNAi-8, OsFPFL4-RNAi transgenic lines 
modulating auxin accumulation in the root. Firstly, the transcript levels of OsFPFL4 were positively correlated with the lateral root number (Fig. $3 \mathrm{~b}$ and e), a process that depends on auxin-mediated establishment. Then, alteration in root auxin levels in OsFPFL4-overexpressing and -RNAi lines were clearly confirmed by the measurement of endogenous IAA content (Fig. 6a). We hypothesize that OsFPFL4 influenced auxin homeostasis in the root by increasing polar auxin transport and/or local auxin biosynthesis, and/or reducing auxin degradation/conjugation. YUCs are evidently key genes for auxin biosynthesis (Zhao et al. 2001), and overexpression of YUCs led to the overproduction of auxin, whereas disruption of YUCs caused developmental defects in roots (Peret et al. 2009). Influx and efflux transporters mediated polar auxin transport, which controlled plant root development (Blilou et al. 2005; Peret et al. 2012). AUX/ $L A X$ loss-of-function mutations led to reduced lateral root formation by affecting lateral root initiation and/or emergence (Marchant et al. 2002; Swarup et al. 2008; Zhao et al. 2015). As a group of early auxin-responsive genes, the GH3 family encodes IAA-amido synthetases that prevent free IAA accumulation (Du et al. 2012). In our study, we found that OsFPFL4 changed the transcript levels of OsYUCs, OsPINs/OsAUXs, and OsGH3s (Fig. 6c-f), which could be one of the reasons why auxin content was altered in OsFPFL4 transgenic plants (Fig. 6a and b). Therefore, our data support that OsFPFL4 modulates lateral root formation in rice by altering auxin accumulation in the root.

It has been reported that the ROS and auxin pathways can extensively impact each other (Kwak et al. 2006). Auxin-induced ROS as signals are directly involved in cell-wall loosening and cell elongation as well as auxinmediated developmental processes (Schopfer 2001; Xia et al. 2015), and cellular redox status is an intrinsic regulator of the plant cell cycle (Diaz Vivancos et al. 2010). Meanwhile, $\mathrm{H}_{2} \mathrm{O}_{2}$ can regulate the root system architecture by modulating the polar transport and redistribution of auxin (Su et al. 2016), and asymmetric ROS accumulation mediates auxin-regulated root gravitropism (Joo et al. 2001). More specifically, root elongation is reduced by ROS via enhancing Rbohs expression, whereas silencing of RbohC accelerates root elongation (Zhang et al. 2014a). Actually, increased ROS may alter auxin signalling through oxidative inactivation or degradation of auxin, and also by the decreased expression of genes involved in auxin signalling and polar auxin transport (Blomster et al. 2011; Peer et al. 2013). For example, when plants are exposed to environmental stresses, ROS can attenuate auxin signalling, leading to altered plant growth and acclimation. In our study, OsFPFL4 overexpression lines, which had more free IAA than wild type, exhibited slightly increased ROS accumulation (Fig. 6a and b; Fig. 7b and c). Although the mRNA levels of Rbohs were reduced in overexpression lines, the expression of multiple genes encoding PERs, which also catalyze ROS production, was increased (Fig. 7d-k; Additional file 2: Table S2). In RNAi lines that had less free IAA accumulation, ROS levels were significantly increased, accompanied by elevated expression of Rbohs (Figs. 6a, 7b-k). The mechanism of auxin-induced ROS production has been pursued. Recently, great progress has been achieved in the molecular link between auxin- and ROS-mediated developmental program. In Arabidopsis, the bHLH transcription factor RSL4 (ROOT HAIR DEFECTIVE 6 LIKE 4) was activated by ARFs which binded to RSL4 promoter on Aux-RE sites (Pires et al. 2013; Mangano et al. 2017). Auxin-ARF activation of RSL4 promoted ROS production by directly regulating the expression of RbohC, RbohJ and several PERs (Hwang et al. 2017; Mangano et al. 2017), suggesting that auxininduced ROS-mediated developmental program is finetuned by the master regulator, RSL4. To elucidate the underlying mechanism that OsFPFL4 orchestrates root and flower development in response to these key developmental signals, ROS and auxin, it is crucial to find transcription factors that function like RSL4 in rice.

\section{Conclusions}

Collectively, our results showed that OsFPFL4 is invovled in the regulation of root and flower development through controlling auxin as well as ROS homeostasis. Specifically, auxin-mediated ROS production might play a role in regulating redox status, which, in turn, modulates auxin homeostasis and signaling, to control plant growth and development (Fig. 10). Thus, ROS production and associated redox processing are an integral part of hormone regulation and function in the control of plant growth and development.

\section{Materials and Methods}

\section{Plant Materials and Growth Conditions}

The japonica rice (Oryza sativa L) variety 'Nipponbare' was used for physiological experiments and genetic transformation in this study. For phenotypic observations, rice seeds were surface sterilized with ethanol $(75 \%, v / v)$ and diluted $\mathrm{NaClO}(1: 3, \mathrm{v} / \mathrm{v})$, followed by thorough rinse with sterilized water, and then were germinated in $1 / 2 \mathrm{MS}$ medium (Murashige and Skoog 1962). Seedlings were grown in a growth chamber at $30^{\circ} \mathrm{C}$ with a 14-h photoperiod and a light intensity of $300 \mu \mathrm{mol}$ photons $\mathrm{m}^{-2} \mathrm{~s}^{-1}$. Relative humidity was controlled at approximately $60 \%$.

\section{Phytohormone and Abiotic Stress Treatments}

Investigation of the responses to phytohormone and abiotic stress treatments was performed according to the described methods (Puig et al. 2013), with minor modifications. Ten-day-old seedlings were treated with $20 \mu \mathrm{M}$ IAA, $200 \mathrm{mM} \mathrm{NaCl}, 100 \mathrm{mM}$ mannitol, 20\% PEG6000, 


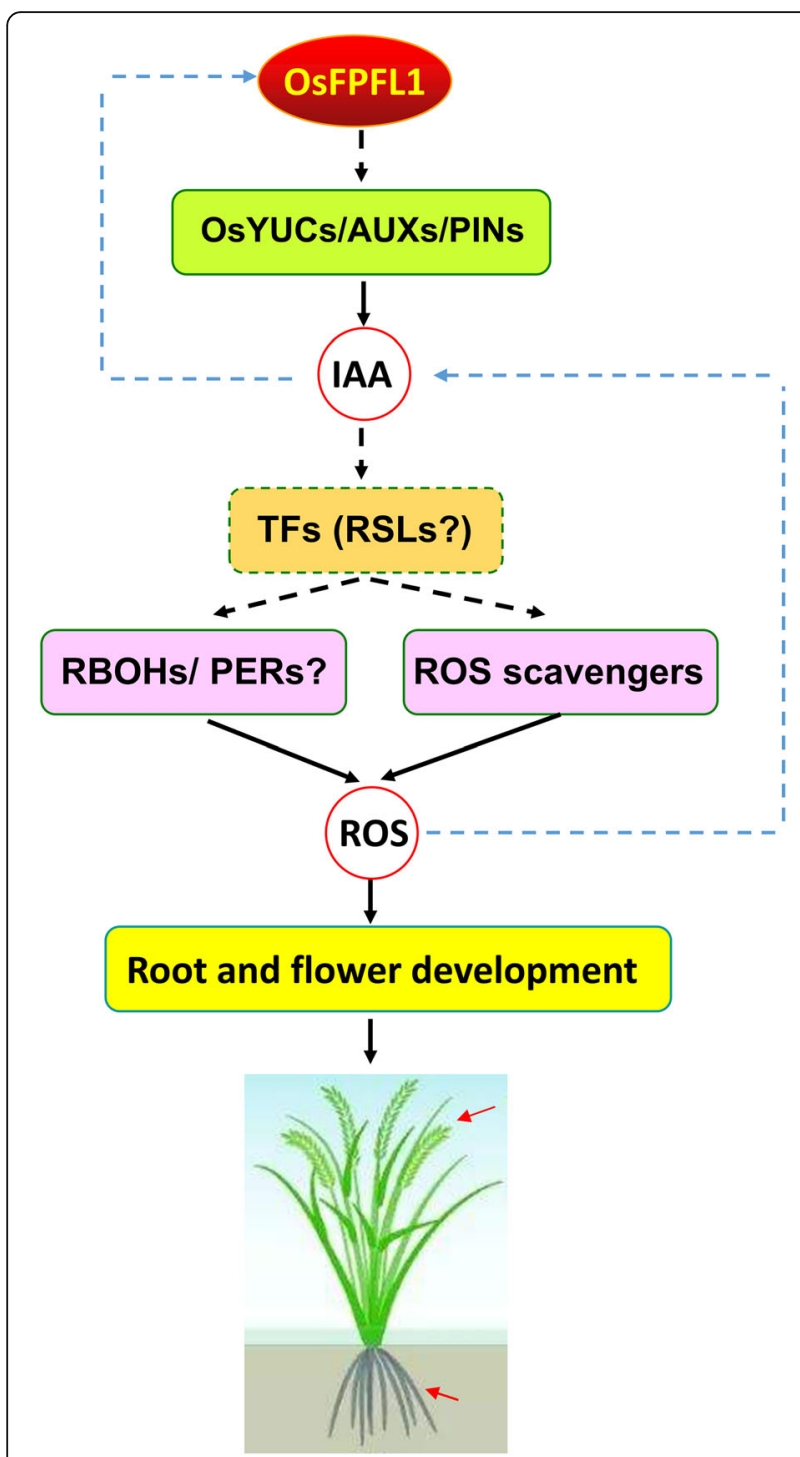

Fig. 10 Working model describing OSFPFL4 controls the root and flower development via affecting auxin as well as ROS homeostasis. OsFPFL4 controls auxin as well as ROS homeostasis via affecting the transcription of genes for auxin biosynthesis and/or transport, and ROS producing and/or scavenging. Auxin-mediated ROS production might play a role in regulating redox status by RSL4 analogs.

Alternatively, ROS control plant growth and development potentially through regulating auxin homeostasis

$42^{\circ} \mathrm{C}$ and $4{ }^{\circ} \mathrm{C}$, respectively. Roots of these seedlings were sampled to evaluate the expression of OsFPFL4.

Vector Construction and Generation of Transgenic Plants For the overexpression construct, the full-length cDNA of OsFPFL4 was amplified and cloned into the modified pCAMBIA1301 vector via BamHI and Pst I and driven by $35 S$ promoter. For the RNA-silencing construct, a cDNA fragment of OsFPFL4 was cloned into the pENTR/D-TOPO vector (Invitrogen) to get the entry clone pENTR/OsFPFL4. The final RNA-silencing vector,
OsFPFL4-RNAi, was generated by a clonase reaction (Invitrogen) between pENTR/OsFPFL4 and the vector pANDA (Miki and Shimamoto 2004). The constructs were transformed into rice calli by using Agrobacterium tumefaciens-mediated transformation as previously described (Ozawa and Takaiwa 2010). Then the transgenic lines were screened based on the hygromycin resistance, GUS staining and expression levels of OsFPFL4. Primers for vector construction are listed in Additional file 1: Table S1.

\section{Morphological Characterization and Quantification of Root System Traits}

Root morphology was examined in seedlings grown on 1/2 MS agar medium. All visible lateral roots originating from the primary root were counted. Adventitious root length was calculated as the average of the three longest adventitious roots (Yan et al. 2014).

\section{RNA Extraction and Quantitative Real-Time PCR}

Total RNA was extracted from different tissues using TRIzol reagent and used for reverse-transcription. Quantitative real-time PCR analysis of the targeted genes was then performed, using rice $\beta$-actin gene as the internal control. Primer sequences for quantitative real-time PCR analysis are given in Additional file 1: Table S1. Three replica experiments were performed for each analysis.

\section{Determination of IAA Content}

IAA content was determined on a high performance liquid chromatography tandem mass spectrometer (HPLCMS-MS) instrument (AB Sciex QTRAP 6500 , Agilent Technologies) according to the protocol described previously (You et al. 2016). Fresh shoot or root samples (300 $\mathrm{mg}$ ) from 10-day-old seedlings grown on 1/2 MS medium were collected and used for IAA content measurement.

\section{ROS Assays}

We used nitroblue tetrazolium (NBT) staining to detect $\mathrm{O}_{2}{ }^{-}$, and 3, 3'-diaminobenzidine (DAB) staining for $\mathrm{H}_{2} \mathrm{O}_{2}$, as described previously (Zhang et al. 2014a). $\mathrm{H}_{2} \mathrm{O}_{2}$ quantification was performed according to the method as described previously (Zhang et al. 2014b).

\section{Pollen Viability Assay}

Evaluation of pollen grain viability was performed as previously described (Shi et al. 2015). Anthers from mature spikelets were crushed and stained in $1 \% \mathrm{I}_{2}-\mathrm{KI}$ solution for $5 \mathrm{~min}$, and then the pollens were observed and photographed under a light microscope using bright-field illumination. The frequency of darkly stained pollen grains was determined from at least 10 plants of each line. 


\section{Supplementary information}

Supplementary information accompanies this paper at https://doi.org/10. 1186/s12284-019-0364-0

Additional file 1: Table S1. Primer sequences used in this study. Additional file 2: Table S2. DEGs (differentially expressed genes) involved in ROS homeostasis in OSFPFL4 transgenic plants.

Additional file 3: Figure S1. FPF1-like proteins in rice.

\section{Abbreviations}

DAB: 3, 3'-diaminobenzidine; NBT: Nitroblue tetrazolium; ROS: Reactive oxygen species

\section{Acknowledgements}

Not applicable.

\section{Authors' Contributions}

HJ conceived and designed the research. GY and WQ conducted experiments. $X Z$ and $Y B$ contributed analytical tools. ZR and MQ prepared seed materials. GY and WQ wrote the manuscript. HJ edited the manuscript. All authors read and approved the manuscript for publication.

\section{Funding}

Funding for this work was provided by National Natural Science Foundation of China $(31771747,31271685)$

\section{Availability of Data and Materials}

The datasets used or analysed in this study are included in the article and its additional files.

\section{Ethics Approval and Consent to Participate}

Not applicable.

\section{Consent for Publication}

Not applicable.

\section{Competing Interests}

The authors declare that they have no competing interests.

Received: 5 June 2019 Accepted: 23 December 2019

Published online: 07 January 2020

\section{References}

Benkova E, Michniewicz M, Sauer M, Teichmann T, Seifertova D, Jurgens G, Friml $J$ (2003) Local, efflux-dependent auxin gradients as a common module for plant organ formation. Cell 115:591-602

Blilou I, Xu J, Wildwater M, Willemsen V, Paponov I, Friml J, Heidstra R, Aida M, Palme K, Scheres B (2005) The PIN auxin efflux facilitator network controls growth and patterning in Arabidopsis roots. Nature 433:39-44

Blomster T, Salojarvi J, Sipari N, Brosche M, Ahlfors R, Keinanen M, Overmyer K, Kangasjarvi J (2011) Apoplastic reactive oxygen species transiently decrease auxin signaling and cause stress-induced morphogenic response in Arabidopsis. Plant Physiol 157:1866-1883

Chen X, Xia X, Guo X, Zhou Y, Shi K, Zhou J, Yu J (2016) Apoplastic $\mathrm{H}_{2} \mathrm{O}_{2}$ plays a critical role in axillary bud outgrowth by altering auxin and cytokinin homeostasis in tomato plants. New Phytol 211:1266-1278

Coudert Y, Perin C, Courtois B, Khong NG, Gantet P (2010) Genetic control of root development in rice, the model cereal. Trends Plant Sci 15:219-226

De Smet I, Vanneste S, Inze D, Beeckman T (2006) Lateral root initiation or the birth of a new meristem. Plant Mol Biol 60:871-887

Diaz Vivancos P, Dong Y, Ziegler K, Markovic J, Pallardo FV, Pellny TK, Verrier PJ, Foyer $\mathrm{CH}$ (2010) Recruitment of glutathione into the nucleus during cell proliferation adjusts whole-cell redox homeostasis in Arabidopsis thaliana and lowers the oxidative defence shield. Plant J 64:825-838

Du H, Wu N, Fu J, Wang S, Li X, Xiao J, Xiong L (2012) A GH3 family member, OsGH3-2, modulates auxin and abscisic acid levels and differentially affects drought and cold tolerance in rice. J Exp Bot 63:6467-6480

Fukaki H, Tasaka M (2009) Hormone interactions during lateral root formation. Plant Mol Biol 69:437-449
Ge L, Chen H, Jiang J, Zhao Y, Xu M, Xu Y, Tan K, Xu Z, Chong K (2004) Overexpression of OsRAA1 causes pleiotropic phenotypes in transgenic rice plants, including altered leaf, flower, and root development and root response to gravity. Plant Physiol 135:1502-1513

Guseman JM, Hellmuth A, Lanctot A, Feldman TP, Moss BL, Klavins E, Villalobos LIAC, Nemhauser JL (2015) Auxin-induced degradation dynamics set the pace for lateral root development. Development 142:905-909

Heyman J, Cools T, Vandenbussche F, Heyndrickx KS, Van Leene J, Vercauteren I, Vanderauwera S, Vandepoele K, De Jaeger G, Van Der Straeten D, De Veylder $L$ (2013) ERF115 controls root quiescent center cell division and stem cell replenishment. Science 342:860-863

Hochholdinger F, Park WJ, Sauer M, Woll K (2004) From weeds to crops: genetic analysis of root development in cereals. Trends Plant Sci 9:42-48

Huang G, Liang W, Sturrock CJ, Pandey BK, Giri J, Mairhofer S, Wang D, Muller L, Tan H, York LM, Yang J, Song Y, Kim Y-J, Qiao Y, Xu J, Kepinski S, Bennett MJ, Zhang D (2018) Rice actin binding protein RMD controls crown root angle in response to external phosphate. Nat Commun 9:2346

Hwang Y, Choi H-S, Cho H-M, Cho H-T (2017) Tracheophytes contain conserved orthologs of a basic helix-loop-helix transcription factor that modulate ROOT HAIR SPECIFIC genes. Plant Cell 29:39-53

Ivanchenko MG, den Os D, Monshausen GB, Dubrovsky JG, Bednarova A, Krishnan N (2013) Auxin increases the hydrogen peroxide $\left(\mathrm{H}_{2} \mathrm{O}_{2}\right)$ concentration in tomato (Solanum lycopersicum) root tips while inhibiting root growth. Ann Bot 112:1107-1116

Jiang M, Zhang J (2003) Cross-talk between calcium and reactive oxygen species originated from NADPH oxidase in abscisic acid-induced antioxidant defence in leaves of maize seedlings. Plant Cell Environ 26:929-939

Jiang W, Zhou S, Zhang Q, Song H, Zhou D-X, Zhao Y (2017) Transcriptional regulatory network of WOX11 is involved in the control of crown root development, cytokinin signals, and redox in rice. J Exp Bot 68:2787-2798

Joo JH, Bae YS, Lee JS (2001) Role of auxin-induced reactive oxygen species in root gravitropism. Plant Physiol 126:1055-1060

Kadota Y, Shirasu K, Zipfel C (2015) Regulation of the NADPH oxidase RBOHD during plant immunity. Plant Cell Physiol 56:1472-1480

Kania T, Russenberger D, Peng S, Apel K, Melzer S (1997) FPF1 promotes flowering in Arabidopsis. Plant Cell 9:1327-1338

Kwak JM, Nguyen V, Schroeder JI (2006) The role of reactive oxygen species in hormonal responses. Plant Physiol 141:323-329

Lavenus J, Goh T, Roberts I, Guyomarc'h S, Lucas M, De Smet I, Fukaki H, Beeckman T, Bennett M, Laplaze L (2013) Lateral root development in Arabidopsis: fifty shades of auxin. Trends Plant Sci 18:455-463

Liu Y, Wang R, Zhang P, Chen Q, Luo Q, Zhu Y, Xu J (2016) The nitrification inhibitor methyl 3-(4-hydroxyphenyl)propionate modulates root development by interfering with auxin signaling via the NO/ROS pathway. Plant Physiol 171:1686-1703

Mangano S, Paola Denita-Juarez S, Choi H-S, Marzol E, Hwang Y, Ranocha P, Melina Velasquez S, Borassi C, Laura Barberini M. Alejandro Aptekmann A, Prometeo Muschietti J, Daniel Nadra A, Dunand C, Cho H-T, Manuel Estevez J (2017) Molecular link between auxin and ROS-mediated polar growth. Proc Natl Acad Sci U S A 114:5289-5294

Marchant A, Bhalerao R, Casimiro I, Eklof J, Casero PJ, Bennett M, Sandberg G (2002) AUX1 promotes lateral root formation by facilitating indole-3-acetic acid distribution between sink and source tissues in the Arabidopsis seedling. Plant Cell 14:589-597

Marin E, Jouannet V, Herz A, Lokerse AS, Weijers D, Vaucheret $H$ Nussaume L, Crespi MD, Maizel A (2010) miR390, Arabidopsis TAS3 tasiRNAs, and their AUXIN RESPONSE FACTOR targets define an autoregulatory network quantitatively regulating lateral root growth. Plant Cell 22:1104-1117

Melzer S, Kampmann G, Chandler J, Apel K (1999) FPF1 modulates the competence to flowering in Arabidopsis. Plant J 18:395-405

Melzer S, Majewski DM, Apel K (1990) Early changes in gene expression during the transition from vegetative to generative growth in the long-day plant Sinapis alba. Plant Cell 2:953-961

Miki D, Shimamoto K (2004) Simple RNAi vectors for stable and transient suppression of gene function in rice. Plant Cell Physiol 45:490-495

Montiel J, Arthikala M-K, Quinto C (2013) Phaseolus vulgaris RbohB functions in lateral root development. Plant Signal Behav 8:e22694

Mueller K, Carstens AC, Linkies A, Angel Torres M, Leubner-Metzger G (2009) The NADPH-oxidase AtrbohB plays a role in Arabidopsis seed after-ripening. New Phytol 184:885-897 
Murashige T, Skoog F (1962) A revised medium for rapid growth and bio assays with tobacco tissue cultures. Physiol Plant 15:473-497

Orman-Ligeza B, Parizot B, de Rycke R, Fernandez A, Himschoot E, Van Breusegem F, Bennett MJ, Perilleux C, Beeckman T, Draye X (2016) RBOHmediated ROS production facilitates lateral root emergence in Arabidopsis. Development 143:3328-3339

Ozawa K, Takaiwa F (2010) Highly efficient agrobacterium-mediated transformation of suspension-cultured cell clusters of rice (Oryza sativa L.) Plant Sci 179:333-337

Parizot B, Laplaze L, Ricaud L, Boucheron-Dubuisson E, Bayle V, Bonke M, De Smet I, Poethig SR, Helariutta Y, Haseloff J, Chriqui D, Beeckman T, Nussaume $L$ (2008) Diarch symmetry of the vascular bundle in Arabidopsis root encompasses the pericycle and is reflected in distich lateral root initiation. Plant Physiol 146:140-148

Passardi F, Penel C, Dunand C (2004) Performing the paradoxical: how plant peroxidases modify the cell wall. Trends Plant Sci 9:534-540

Peer WA, Cheng Y, Murphy AS (2013) Evidence of oxidative attenuation of auxin signalling. J Exp Bot 64:2629-2639

Peret B, De Rybel B, Casimiro I, Benkova E, Swarup R, Laplaze L, Beeckman T, Bennett MJ (2009) Arabidopsis lateral root development: an emerging story. Trends Plant Sci 14:399-408

Peret B, Swarup K, Ferguson A, Seth M, Yang Y, Dhondt S, James N, Casimiro I, Perry P, Syed A, Yang H, Reemmer J, Venison E, Howells C, Perez-Amador MA, Yun J, Alonso J, Beemster GTS, Laplaze L, Murphy A, Bennett MJ, Nielsen E, Swarup R (2012) AUX/LAX genes encode a family of auxin influx transporters that perform distinct functions during Arabidopsis development. Plant Cell 24:2874-2885

Petricka JJ, Winter CM, Benfey PN (2012) Control of Arabidopsis doot development. In: Merchant SS (ed) Annual review of plant biology, Vol 63. Annual review of plant biology, pp 563-590

Pignocchi C, Fletcher JM, Wilkinson JE, Barnes JD, Foyer CH (2003) The function of ascorbate oxidase in tobacco. Plant Physiol 132:1631-1641

Pires ND, Yi K, Breuninger H, Catarino B, Menand B, Dolan L (2013) Recruitment and remodeling of an ancient gene regulatory network during land plant evolution. Proc Natl Acad Sci U S A 110:9571-9576

Potocky M, Jones MA, Bezvoda R, Smirnoff N, Zarsky V (2007) Reactive oxygen species produced by NADPH oxidase are involved in pollen tube growth. New Phytol 174:742-751

Puig J, Meynard D, Khong GN, Pauluzzi G, Guiderdoni E, Gantet P (2013) Analysis of the expression of the AGL17-like clade of MADS-box transcription factors in rice. Gene Expr Patterns 13:160-170

Qi J, Wang J, Gong Z, Zhou J-M (2017) Apoplastic ROS signaling in plant immunity. Curr Opin Plant Biol 38:92-100

Schopfer P (2001) Hydroxyl radical-induced cell-wall loosening in vitro and in vivo: implications for the control of elongation growth. Plant J 28:679-688

Shi X, Sun X, Zhang Z, Feng D, Zhang Q, Han L, Wu J, Lu T (2015) GLUCAN SYNT HASE-LIKE 5 (GSL5) plays an essential role in male fertility by regulating callose metabolism during microsporogenesis in rice. Plant Cell Physiol 56: 497-509

Smykal P, Gleissner R, Corbesier L, Apel K, Melzer S (2004) Modulation of flowering responses in different Nicotiana varieties. Plant Mol Biol 55:253-262

Su C, Liu L, Liu H, Ferguson BJ, Zou Y, Zhao Y, Wang T, Wang Y, Li X (2016) $\mathrm{H}_{2} \mathrm{O}_{2}$ regulates root system architecture by modulating the polar transport and redistribution of auxin. J Plant Biol 59:260-270

Sundaravelpandian K, Chandrika NNP, Schmidt W (2013) PFT1, a transcriptional mediator complex subunit, controls root hair differentiation through reactive oxygen species (ROS) distribution in Arabidopsis. New Phytol 197:151-161

Swarup K, Benkova E, Swarup R, Casimiro I, Peret B, Yang Y, Parry G, Nielsen E, De Smet I, Vanneste S, Levesque MP, Carrier D, James N, Calvo V, Ljung K, Kramer E, Roberts R, Graham N, Marillonnet S, Patel K, Jones JDG, Taylor CG, Schachtman DP, May S, Sandberg G, Benfey P, Friml J, Kerr I, Beeckman T, Laplaze L, Bennett MJ (2008) The auxin influx carrier LAX3 promotes lateral root emergence. Nat Cell Biol 10:946-954

Tsukagoshi H, Busch W, Benfey PN (2010) Transcriptional regulation of ROS controls transition from proliferation to differentiation in the root. Cell 143: 606-616

Van Norman JM, Xuan W, Beeckman T, Benfey PN (2013) To branch or not to branch: the role of pre-patterning in lateral root formation. Development 140:4301-4310
Wang X, Fan S, Song M, Pang C, Wei H, Yu J, Ma Q, Yu S (2014) Upland cotton gene GhFPF1 confers promotion of flowering time and shade-avoidance responses in Arabidopsis thaliana. PLoS One 9:e91869

Xia X, Wang Y, Zhou Y, Tao Y, Mao W, Shi K, Asami T, Chen Z, Yu J (2009) Reactive oxygen species are involved in brassinosteroid- induced stress tolerance in cucumber. Plant Physiol 150:801-814

Xia X, Zhou Y, Shi K, Zhou J, Foyer C, Yu J (2015) Interplay between reactive oxygen species and hormones in the control of plant development and stress tolerance. J Exp Bot 66:2839-2856

Xu M, Jiang J, Ge L, Xu Y, Chen H, Zhao Y, Bi Y, Wen J, Chong K (2005) FPF1 transgene leads to altered flowering time and root development in rice. Plant Cell Rep 24:79-85

Yan Y, Wang H, Hamera S, Chen X, Fang R (2014) miR444a has multiple functions in the rice nitrate-signaling pathway. Plant J 78:44-55

Yang L, Zhang J, He JN, Qin YY, Hua DP, Duan Y, Chen ZZ, Gong ZZ (2014) ABAmediated ROS inmitochondria regulate root meristem activity by controlling PLETHORA expression in Arabidopsis. PLoS Genet 10:e1004791

Yoon EK, Kim J-W, Yang JH, Kim S-H, Lim J, Lee WS (2014) A molecular framework for the differential responses of primary and lateral roots to auxin in Arabidopsis thaliana. J Plant Biol 57:274-281

You C, Zhu H, Xu B, Huang W, Wang S, Ding Y, Liu Z, Li G, Chen L, Ding C, Tang $S$ (2016) Effect of removing superior spikelets on grain filling of inferior spikelets in rice. Front Plant Sci 7:1161

Yu L, Wu J, Zhang Z, Miao Z, Zhao P, Wang Z, Xiang C (2017) Arabidopsis MADSbox transcription factor AGL21 acts as environmental surveillance of seed germination by regulating ABI5 expression. Mol Plant 10:834-845

Zhang C, Bousquet A, Harris JM (2014a) Abscisic acid and LATERAL ROOT ORGAN DEFECTIVE/NUMEROUS INFECTIONS AND POLYPHENOLICS modulate root elongation via reactive oxygen species in Medicago truncatula. Plant Physiol 166:644-U295

Zhang G, Xu N, Chen H, Wang G, Huang J (2018) OsMADS25 regulates root system development via auxin signalling in rice. Plant J 95:1004-1022

Zhang H, Liu Y, Wen F, Yao D, Wang L, Guo J, Ni L, Zhang A, Tan M, Jiang M (2014b) A novel rice $\mathrm{C}_{2} \mathrm{H}_{2}$-type zinc finger protein, ZFP36, is a key player involved in abscisic acid-induced antioxidant defence and oxidative stress tolerance in rice. J Exp Bot 65:5795-5809

Zhang HM, Forde BG (1998) An Arabidopsis MADS box gene that controls nutrient-induced changes in root architecture. Science 279:407-409

Zhao H, Ma T, Wang X, Deng Y, Ma H, Zhang R, Zhao J (2015) OsAUX1 controls lateral root initiation in rice (Oryza sativaL.). Plant Cell Environ 38:2208-2222

Zhao YD, Christensen SK, Fankhauser C, Cashman JR, Cohen JD, Weigel D, Chory $J$ (2001) A role for flavin monooxygenase-like enzymes in auxin biosynthesis. Science 291:306-309

\section{Publisher's Note}

Springer Nature remains neutral with regard to jurisdictional claims in published maps and institutional affiliations.

\section{Submit your manuscript to a SpringerOpen ${ }^{\circ}$ journal and benefit from:}

- Convenient online submission

- Rigorous peer review

- Open access: articles freely available online

- High visibility within the field

- Retaining the copyright to your article

Submit your next manuscript at $>$ springeropen.com 